\title{
The anatomy of Meyer's loop revisited: changing the anatomical paradigm of the temporal loop based on evidence from fiber microdissection
}

\author{
Cristina Goga, MD, 1,2 and Uğur Türe, MD1 \\ 1Department of Neurosurgery, Yeditepe University School of Medicine, Istanbul, Turkey; and 'Department of Anatomy, University \\ of Medicine and Pharmacy Targu Mures, Romania
}

\begin{abstract}
OBJECT The goal in this study was to explore and further refine comprehension of the anatomical features of the temporal loop, known as Meyer's loop.

METHODS The lateral and inferior aspects of 20 previously frozen, formalin-fixed human brains were dissected under the operating microscope by using fiber microdissection.

RESULTS A loop of the fibers in the anterior temporal region was clearly demonstrated in all dissections. This temporal loop, or Meyer's loop, is commonly known as the anterior portion of the optic radiation. Fiber microdissection in this study, however, revealed that various projection fibers that emerge from the sublentiform portion of the internal capsule (IC-SL), which are the temporopontine fibers, occipitopontine fibers, and the posterior thalamic peduncle (which includes the optic radiation), participate in this temporal loop and become a part of the sagittal stratum. No individual optic radiation fibers could be differentiated in the temporal loop. The dissections also disclosed that the anterior extension and angulation of the temporal loop vary significantly.
\end{abstract}

CONCLUSIONS The fiber microdissection technique provides clear evidence that a loop in the anterior temporal region exists, but that this temporal loop is not formed exclusively by the optic radiation. Various projection fibers of the IC-SL, of which the optic radiation is only one of the several components, display this common course. The inherent limitations of the fiber dissection technique preclude accurate differentiation among individual fibers of the temporal loop, such as the optic radiation fibers.

http://thejns.org/doi/abs/10.3171/2014.12.JNS14281

KEY WORDS fiber dissection technique; Meyer's loop; microsurgical anatomy; optic radiation; temporal loop; white matter anatomy

$\mathrm{T}$ HE ultimate anatomical details of the optic radiation, particularly the temporal loop of the anterior optic radiation fibers commonly known as Meyer's loop, continue to elude us. This ambiguity persists because, in humans, contemporary histological staining techniques, which distinguish degenerated and nondegenerated fibers, and brain dissection techniques have so far been unable to indisputably demonstrate and comprehensively illustrate the definite course of individual optic radiation fibers, which originate from the lateral geniculate body and terminate in the occipital visual cortex. Applying his- tological degeneration staining techniques, in 1907 Adolf Meyer (Fig. 1) defined the first detailed configuration of the optic radiation, according to which the anterior fibers of the optic radiation detour from the lateral geniculate body into the temporal region before reaching the occipital cortex..$^{25,54}$ The myelin-stained anterior fibers of the optic radiation are visible within the temporal lobe in his serial coronal brain sections, but neither the term "temporal loop" nor "Meyer's loop" was mentioned in his work. Serial brain sections are an inadequate method to evaluate and reconstruct the course of these temporal optic fibers.

ABBREVIATIONS $A C=$ anterior commissure; $I C=$ internal capsule; $I C-S L=$ sublentiform portion of the internal capsule; $S S=$ sagittal stratum .

SUBMITTED February 5, 2014. ACCEPTED December 1, 2014.

INCLUDE WHEN CITING Published online January 30, 2015; DOI: 10.3171/2014.12.JNS14281.

DISCLOSURE This project was partly supported by the Sectorial Operational Programme Human Resources Development (SOP HRD), financed from the European Social Fund and by the Romanian Government under the contract number POSDRU 80641 through a grant given to Dr. Goga in 2012 and 2013. 


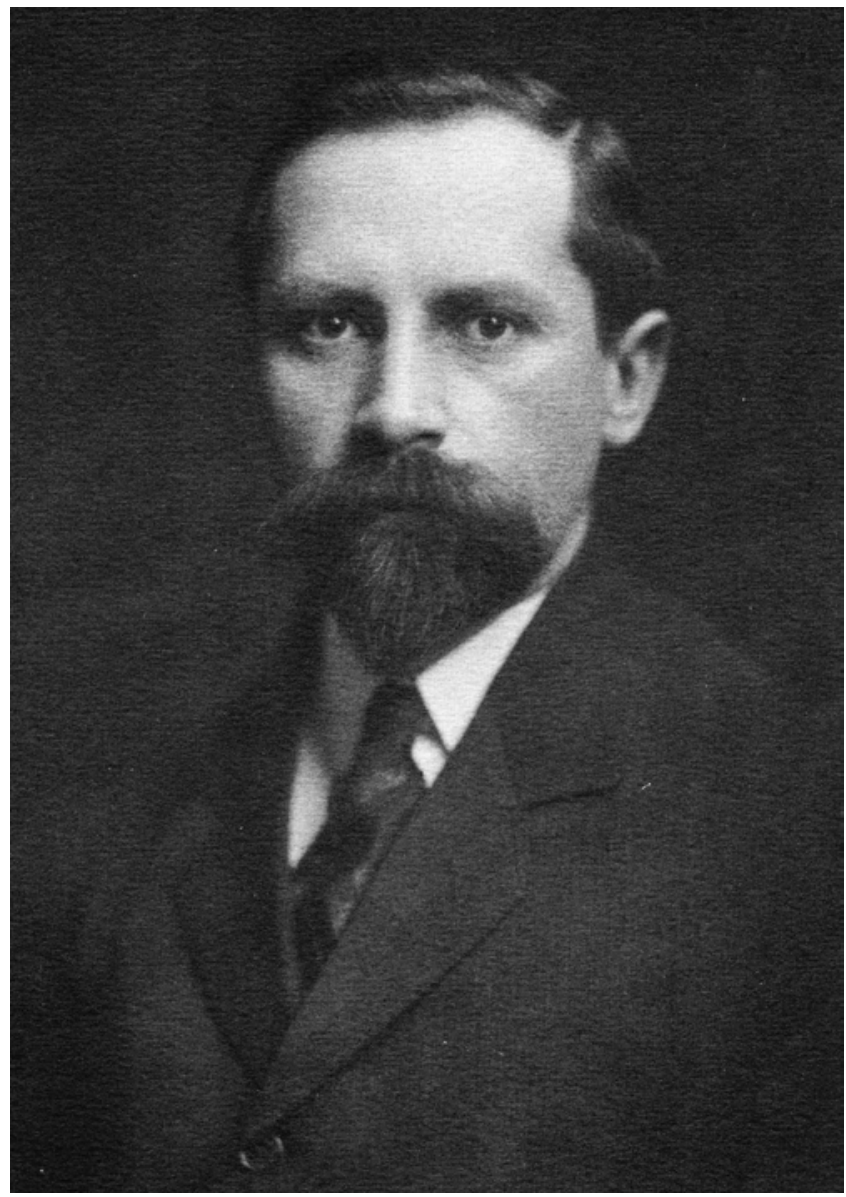

FIG. 1. Adolf Meyer (1866-1950), Professor of Psychiatry at Johns Hopkins School of Medicine in Baltimore, Maryland, was an influential psychiatrist, neurologist, neuropathologist, and neuroanatomist. He provided the first detailed configuration that asserted the peculiar detour into the temporal lobe of the anterior part of the optic radiation. Reprinted from Winters E (ed): The Collected Papers of Adolf Meyer, Vol. 1 Neurology. Baltimore: The Johns Hopkins Press, 1950.

Therefore the existence and anatomical features, such as composition and location of the supposed temporal loop, had not been clarified at that time.

The first reconstruction of Meyer's configuration of the optic radiation was an artistic diagram published by Cushing to support his clinical correlations of visual field defects with temporal lobe tumors. ${ }^{5}$ This diagram illustrates the peculiar temporal detour of the optic radiation that became associated with the term "temporal loop," and later "Meyer's loop." The popularization of this configuration and its perpetuation through reproduction of these diagrams in standard anatomical texts seem to have given finality to the regional anatomy. Although we are fascinated by the logic of this diagrammatic representation, this actually oversimplifies one of the most complex fiber systems, the projection fibers of the temporal and occipital regions, one component of which is the optic radiation. The true structure and course of the temporal and occipital projection fibers, such as temporopontine fibers, occipitopontine fibers, and posterior thalamic peduncle remain obscure even today.

Recent studies in which the fiber dissection technique has been applied claim to have demonstrated the optic radiation and, therefore, support the paradigm that the temporal loop is composed exclusively of visual projection fibers. ${ }^{2,3,4,6,10,22,28-30,34,44,46,47}$ However, close analysis of their supporting illustrations fails to demonstrate unequivocally the course of individual fibers from their origin in the lateral geniculate body to their termination in the visual occipital cortex. To understand the anatomical concept of the temporal loop and engender a realistic appreciation of the anatomical information that can be inferred from fiber microdissection, we revisited the anatomy of the temporal loop. The intention of this report is to explore and attempt to clarify the ambiguities that surround this anatomical structure.

\section{Methods}

Twenty formaldehyde-fixed and previously frozen human brain hemispheres were dissected and studied under the operating microscope by applying the fiber microdissection technique, which has been extensively described. ${ }^{14-16,21,51,52,56}$ The achievement of skillful and meticulous microtechniques during fiber microdissection to reveal the most minute fibers requires certain tools: an operating microscope, fine watchmaker's forceps, and a pressure-regulated surgical suction system. Dissection always began at the lateral and inferior aspects and proceeded in a stepwise manner, according to the method recommended by the senior author (U.T.). ${ }^{51,52,56}$ The anatomy of every progressive stage of the dissection was photographed (Nikon D7000 camera; AF-S VR MicroNikkor 105 mm f/2.8 G IF-ED lens, Nikon Corp.).

\section{Results}

\section{Dissection of the Lateral Aspect of the Cerebral Hemisphere}

Dissection involves the lateral aspect of the temporal and occipital regions of the cerebral hemisphere. The cerebral cortex and $U$ fibers are removed to reveal the inferior aspect of the superior longitudinal fasciculus around the insula (Fig. 2A). Removal of the insular cortex, extreme capsule, claustrum, and the external capsule reveals the putamen and the corona radiata at its periphery (Fig. 2B). The basal surface of the putamen is covered by 2 long association fiber systems, the uncinate fasciculus and the occipitofrontal fasciculus, which display a parallel course in this region, and are in fact compacted to a single bundle without a clear demarcation. As they advance into the temporal lobe, both fiber systems fan out and follow divergent courses. The uncinate fasciculus turns anteriorly to reach the temporal pole region and displays a typical Cshaped configuration, while the occipitofrontal fasciculus maintains its direct, posterior course and then becomes part of the sagittal stratum (SS) to reach temporal and occipital regions. ${ }^{7,50}$ The occipitofrontal fasciculus is, in fact, the lateral component of the SS.

The putamen is removed to reveal the globus pallidus and the internal capsule (IC) in continuity with the corona radiata (Fig. 2C). Dissection of the basal part of the putamen is particularly delicate, to prevent damage to the 

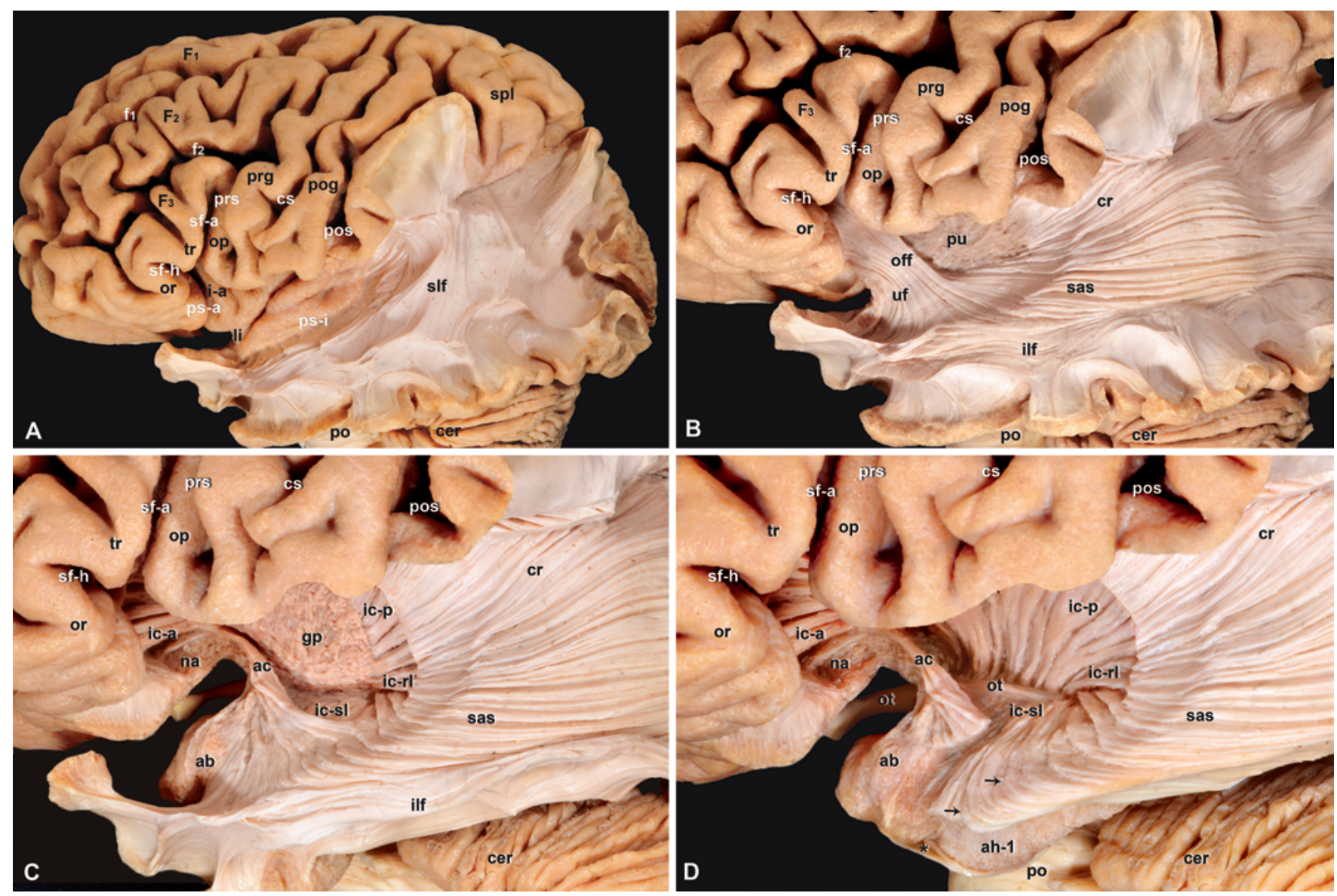

FIG. 2. Photographs of brain specimens showing serial dissections of the lateral aspect of the left cerebral hemisphere. A: The cortex and the $U$ fibers of the temporal and occipital regions are dissected to demonstrate the inferior aspect of the superior longitudinal fasciculus (slf) around the insula. B: Removal of the insular cortex, extreme capsule, claustrum, and external capsule demonstrates the uncinate fasciculus (uf), occipitofrontal fasciculus (off), putamen (pu), corona radiata (cr) and SS (sas). The occipitofrontal fasciculus proceeds into the temporal region and becomes a part of the SS. C: The globus pallidus (gp) with the IC at its periphery becomes visible after removal of the putamen. Dissecting away the uncinate fasciculus and the occipitofrontal fasciculus reveals the lateral extension of the AC (ac) that participates in the formation of the SS by intercrossing the fibers of the IC-SL (ic-sl) in the vicinity of the temporal loop. D: Removing the globus pallidus reveals the temporopontine and occipitopontine projection fibers located in the lateral aspect of the IC-SL passing over the optic tract (ot) to proceed into the temporal loop and then become a part of the SS. The lateral extension of the AC has been severed to fully expose the temporal loop of the projection fibers (arrows) that emerge from the IC-SL. Individual optic radiation fibers are indistinguishable from the various projection fibers that form the temporal loop. The temporal loop of the projection fibers, which includes the optic radiation, is located in the roof of the temporal horn of the lateral ventricle, and becomes fully exposed after removal of the inferior longitudinal fasciculus (ilf). Further dissection in the mediobasal temporal region reveals the basolateral nucleus of amygdala (ab) and the Ammon's horn on the medial aspect of the floor of the temporal horn of the lateral ventricle. Removal of the alveus demonstrates the CA-1 field of Ammon's horn (ah-1). Abbreviations with white letters denote the sulci and fissures. The asterisk (in panel D) denotes the uncal recess of the temporal horn of the lateral ventricle. cer = cerebellum; $c s=$ central sulcus of Rolando; $F_{1}=$ superior frontal gyrus; $F_{2}=$ middle frontal gyrus; $F_{3}=$ inferior frontal gyrus; $f_{1}=$ superior frontal sulcus; $f_{2}=$ inferior frontal sulcus; $i-a=$ insular apex; ic-a $=$ anterior limb of the internal capsule; ic- $p=$ posterior limb of the internal capsule; ic-rl = retrolentiform portion of the internal capsule; $\mid \mathrm{i}=$ limen insula; $n a=$ nucleus accumbens; op = pars opercularis of $F_{3}$; or = pars orbitalis of $F_{3}$; po = pons; pog = postcentral gyrus; pos = postcentral sulcus; $p r g$ = precentral gyrus; $p r s=$ precentral sulcus; $p s-a=$ anterior periinsular sulcus; $p s-i=$ inferior periinsular sulcus; sf-a = ascending ramus of the sylvian fissure; $\mathrm{sf}-\mathrm{h}=$ horizontal ramus of the sylvian fissure; spl = superior parietal lobule; tr $=$ pars triangularis of $\mathrm{F}_{3}$.

lateral extension of the anterior commissure (AC). The lateral extension of the $\mathrm{AC}$ is visible as a compact cylindrical structure covered by a thin sheet (Gratiolet's canal), and is completely revealed after dissecting away the uncinate fasciculus and the occipitofrontal fasciculus. ${ }^{16,31,51}$ Thus the coiled, rope-like arrangement of fibers forming the lateral extension of the AC can be clearly demonstrated. The fibers located in its posterior portion follow an inferior rota- tion and proceed beneath the compact bundle anteriorly, to the temporal pole and amygdala, where they overlap and intermingle with the uncinate fasciculus fibers. The anterior fibers of the lateral extension of the $\mathrm{AC}$ pass above and advance posteriorly into the temporal region, where they intercross with the fibers of the sublentiform portion of the internal capsule (IC-SL) to form the SS. The point of intercrossing between the AC fibers and the projection fibers of 
the IC-SL is located in the vicinity of the temporal loop. The lateral extension of the AC is, therefore, another component that participates, together with the laterally located occipitofrontal fasciculus and the medially located IC-SL, in the formation of the SS.

The IC is the broad white-matter band of projection fibers interposed between the lenticular nucleus (putamen and globus pallidus) on the lateral side and the caudate nucleus and the thalamus on the medial side. ${ }^{41,45,48}$ Based on the orientation of the component fibers, it may be divided into an anterior limb between the lenticular nucleus and the caudate nucleus (lenticulocaudate portion), and a posterior limb between the thalamus and the caudate nucleus. The posterior limb is more complex and may be subdivided into a lenticulothalamic portion, a retrolentiform portion, and IC-SL. The IC-SL is composed of the projection fibers that are located beneath the lenticular nucleus and above the tail of the caudate nucleus.

The projection fibers of all these portions of the IC diverge beyond the limits of the lentiform nucleus into the corona radiata and the SS. Within the corona radiata these projection fibers become intermingled with the commissural fibers of the corpus callosum and, together, follow a joint course, which extends to and from their corresponding cortical areas. ${ }^{41,45,48}$ The equivalent of the corona radiata in the temporal and occipital regions would be the SS. The difference, in these regions, is that the projection fibers of the IC-SL become intermingled with 2 additional components, the commissural fibers of the $\mathrm{AC}$ and the association fibers of the occipitofrontal fasciculus. All of these become interwoven and follow a joint course to the temporal and the occipital regions as the compact SS, and cannot be differentiated during the dissection process. ${ }^{16,18,19,21,25,36,51}$

At this stage, the globus pallidus is carefully removed to completely reveal the IC. Next, the lateral extension of the $\mathrm{AC}$ is severed to expose the IC-SL and the temporal loop (Fig. 2D). The projection fibers of the IC-SL pass under the globus pallidus and over the tail of the caudate nucleus and consist of the following: the temporopontine fibers (Türck's bundle), occipitopontine fibers, and the corticothalamic and thalamocortical fibers of the inferior thalamic peduncle and posterior thalamic peduncle. ${ }^{41,45,48,51,56}$ The temporopontine and occipitopontine projection fibers are located in the lateral aspect of the IC-SL and are revealed when a lateral dissection is performed, whereas an inferior dissection reveals the inferior thalamic peduncle and posterior thalamic peduncle, which are located in the medial aspect of the IC-SL. These temporopontine and occipitopontine projection fibers originate in the pontine nuclei, course in the lateral aspect of the cerebral peduncle, and then continue their course in the IC-SL by passing above the optic tract. In the temporal region, some of the IC-SL fibers proceed anteriorly and course over the uncal recess of the temporal horn to reach the posterolateral aspect of the amygdala or the temporal pole.

At this point in our dissection it became evident that all the projection fibers of the IC-SL that join the SS form a loop posteriorly, after making an initial anterior detour in the temporal region. The most anterior fibers describe the longest detour and the greatest angular loop. This loop of the projection fibers in the anterior temporal region was clearly visible in all specimens. However, we could not distinguish which of these were individual optic radiation fibers in the temporal loop. It is absolutely clear that all projection fibers that emerge from the IC-SL and join the SS pursue this common course in the temporal lobe, and therefore together compose the temporal loop. The transition between the IC-SL and the SS-in other words, the point of intercrossing of all the projection fibers of the ICSL with the AC fibers-can be identified in the vicinity of the temporal loop. This intercrossing of the AC fibers with the IC-SL complicates the dissection of the temporal loop.

Dissecting the inferior longitudinal fasciculus concludes the dissection and demonstrates fully the temporal loop and the SS. The inferior longitudinal fasciculus joins the inferolateral aspect of the SS without a clear demarcation. In the anterior temporal region, the fibers of the inferior longitudinal fasciculus overlap and intercross with the projection fibers of the temporal loop. It is extremely difficult to delineate the fine fibers that participate in the temporal loop from the intercrossing fibers, such as the $\mathrm{AC}$ or the inferior longitudinal fasciculus. Even applying the most proficient fiber microdissection, the tip of the temporal loop could not be accurately delineated. Rather, the boundaries of the loop were established arbitrarily, and we retained most of the fibers participating in the loop intact and sharply severed the intervening fibers at the intercrossing point. For this reason, we were precluded from gathering morphometric data related to the precise location of the temporal loop.

\section{Dissection of the Inferior Aspect of the Cerebral Hemisphere}

Dissection begins in the mediobasal temporal region. The midbrain is severed to achieve adequate visualization of the parahippocampal gyrus and the uncus. The parahippocampal gyrus, the hippocampus, and the fimbria of the fornix are detached at the level of the isthmus of the cingulate gyrus and from between the collateral sulcus and the choroidal fissure. With this maneuver, the entire anatomy of the roof of the temporal horn is demonstrated (Fig. 3A). The choroid plexus is then removed along the choroidal fissure. Careful removal of the ependyma and the subependymal veins from the roof of the temporal horn reveal the stria terminalis, the tail of the caudate nucleus, and the tapetum (Fig. 3B). The tapetum is composed of a subgroup of callosal fibers in the splenial region that arch anteriorly in the roof of the atrium and the temporal horn of the lateral ventricles, and separate the ependyma from the laterally placed SS. The tapetal fibers extend over the whole lateral portion of the roof of the temporal horn, just lateral to the tail of the caudate nucleus.

At this point, removing the stria terminalis and then the tail of the caudate nucleus reveals the medial aspect of IC$\mathrm{SL}$, which is composed of the inferior thalamic peduncle and the posterior thalamic peduncle (Fig. 3C). The inferior thalamic peduncle consists of the temporopulvinar fibers (the temporopulvinar bundle of Arnold), which are the projection fibers that originate in the pulvinar of the thalamus and terminate in the amygdala and the temporal pole. ${ }^{16}$ The posterior thalamic peduncle includes thalamo- 

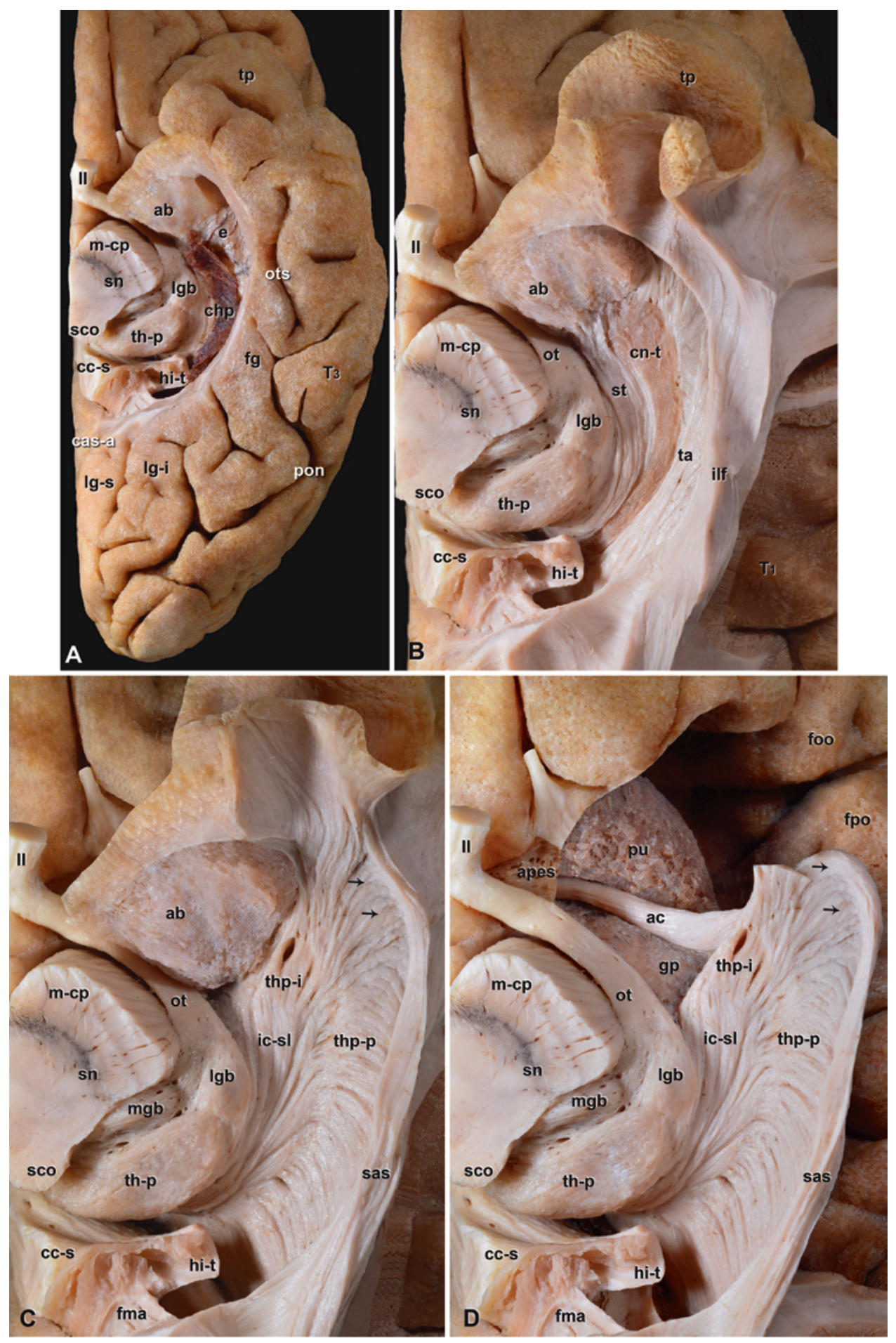

FIG. 3. Photographs of brain specimens showing serial dissections of the inferior aspect of the left cerebral hemisphere. A: The parahippocampal gyrus and hippocampus are removed to expose the roof of the temporal horn of the lateral ventricle covered by the ependyma (e) and choroid plexus (chp). B: Removing the ependyma reveals the stria terminalis (st), the tail of the caudate nucleus (cn-t) and the tapetum of the corpus callosum (ta). Dissecting the cortex and $U$ fibers of the fusiform gyrus and inferior temporal gyrus reveals the inferior longitudinal fasciculus (ilf), which borders the floor of the temporal horn. C: Removal of the stria terminalis and the tail of the caudate nucleus reveals the projection fibers located in the medial aspect of the IC-SL (ic-sl), which are the inferior thalamic peduncle (thp-i) and the posterior thalamic peduncle (thp-p). The inferior thalamic peduncle (itp) that extends as far as the amygdala (ab) and temporal pole (tp) is demonstrated. The temporal loop (arrows) of the posterior thalamic peduncle becomes visible. The posterior thalamic peduncle is one of the several projection fibers of the IC-SL and includes the optic radiation. D: Removal of the amygdala and further dissection at the level of the anterior perforated substance (apes) reveals the AC (ac), globus pallidus (gp) and putamen (pu). Extensive dissection of the inferior aspect of the temporal lobe completely exposes the posterior thalamic peduncle that together with various projection fibers of the IC-SL forms the temporal loop (arrows), and then intercrosses with the AC fibers to become a part of the SS (sas). FIG. 3. (continued) $\rightarrow$ 
FIG. 3. Abbreviations with white letters denote the sulci and fissures. cas-a = anterior calcarine sulcus; $c c-s=$ splenium of the corpus callosum; fg = fusiform gyrus; fma = forceps major (radiation of the corpus callosum); foo = frontoorbital operculum; fpo = frontoparietal operculum; hi-t = tail of hippocampus; II = optic nerve; Ig-i = inferior lingual gyrus; lg-s = superior lingual gyrus; Igb = lateral geniculate body; $\mathrm{m}-\mathrm{cp}=$ cerebral peduncle of midbrain (crus cerebri); mgb = medial geniculate body; ot = optic tract; ots = occipitotemporal sulcus; pon = preoccipital notch; $s c o=$ superior colliculus; $s n=$ substantia nigra; th $-p=$ pulvinar of the thalamus; $T_{1}=$ superior temporal gyrus; $T_{3}=$ inferior temporal gyrus.

cortical and corticothalamic projection fibers that extend between the pulvinar region of the thalamus and the temporal and occipital cortex. Among these projection fibers are the optic and the auditory radiations. In fact, the optic radiation is only one of the components of the posterior thalamic peduncle.

Our dissections clearly show that both the inferior and posterior thalamic peduncle fibers originate in the pulvinar of the thalamus and arch anteriorly in the roof of the temporal horn to advance above the tail of the caudate nucleus. The inferior thalamic peduncle comprises the fibers that extend over the anteromedial portion of the roof of the temporal horn to reach the posterior and lateral aspects of the amygdala and the temporal pole. The posterior thalamic peduncle includes the fibers of the IC-SL that extend from the pulvinar to the anterolateral portion of the roof of the temporal horn, where they curve posteriorly and display a sequence of loops (the temporal loop or Meyer's loop), to become part of the SS. In the roof of the temporal horn, all the projection fibers of the posterior thalamic peduncle, of which the optic radiation is a component part, participate in this temporal loop. Among these fibers, individual fibers that originate in the lateral geniculate body and course in the temporal loop, fibers that would form the true optic radiation, could not be distinguished. It is evident in our dissections that the projection fibers of the posterior thalamic peduncle, visible in the roof of the temporal horn, originate in the pulvinar of the thalamus and not in the lateral geniculate body; therefore these fibers are not optic radiation but various projection fibers to the temporal and occipital regions.

The dissection proceeds at the level of the anterior perforated substance, after the amygdala is removed. The relationship of the amygdala with the ansa peduncularis, the basal ganglia, and the $\mathrm{AC}$ is demonstrated in the process (Fig. 3D). The fibers of the temporal loop together with the AC, as well as the tapetum and the inferior longitudinal fasciculus, are intricately intertwined in the anterior temporal region. Therefore the difficulties pertaining to an accurate delineation of the temporal loop are obvious. The temporal loop is visible in the anterior portion of the roof of the temporal horn, occupying the space between the tail of the caudate nucleus and the collateral eminence, but never reached as far as the amygdala or the uncal recess of the temporal horn. In our dissections the anterior extension and the angulation of the temporal loop varied, but was always located in the anterolateral portion of the roof of the temporal horn, whereas the anteromedial portion of the roof, behind the amygdala, did not contain projection fibers involved in the temporal loop.

\section{Discussion}

The first illustrations of the temporal loop formed of projection fibers appeared in the work on fiber dissection by Johann Christian Reil ${ }^{43}$ in 1807, but this anatomical peculiarity was not described in his publication. In 1854 Louis Pierre Gratiolet and Francois Leuret described the optic radiation, but with indefinite anatomical details. ${ }^{13,20}$ The paradigm that the temporal loop is composed exclusively of visual projection fibers originates from the histological studies of Paul Emil Flechsig in $1896^{11,12}$ and LaSalle Archambault in 1906. ${ }^{1}$ Expanding on these observations in 1907, Adolf Meyer provided evidence that a temporal component of the optic radiation exists. ${ }^{25,54} \mathrm{He}$ analyzed the patterns of fiber degeneration following ischemic and traumatic lesions in myelin-stained, serial, coronal brain sections. This technique is inadequate for unequivocally clarifying the composition, location, and course of the supposed temporal loop because it fails to accurately trace the course of fibers from one brain section to another, and to distinguish the individual optic radiation from the other intricately arranged and intermingling projection fibers. Meyer himself acknowledged that "In the temporal region the auditory radiation to the transverse temporal gyrus and Türck's bundle (temporopontine fibers) to the lateral part of the crus (...) complicate the simple arrangement of the visual fibers of the optic path." 25

To depict the supposed temporal loop, artistic diagrams showing the optic radiation from different views were published in 1922 by Cushing, and became extensively popularized..$^{5}$ These diagrams depict an unrealistic, oversimplified representation of the projection fibers for the temporal and occipital regions, of which the optic radiation is only one of several components. Thus the true location and composition of the temporal loop and the possibility that it might be composed exclusively from the optic radiation fibers remained ambiguous. In addition, the subsequent clinical studies that correlated the postoperative visual field defects following temporal lobe resections with the extent of the resection, an association that could indirectly demonstrate the location of the optic radiation fibers, failed to clarify these ambiguities..$^{9,17,23,26,32,33,49,53}$

\section{Evidence Derived From Fiber Microdissection Supports the Existence of a Temporal Loop Composed of Various Projection Fibers and not Exclusively of the Optic Radiation}

The few fiber dissection studies that followed Meyer's original observations contributed inconsistent results regarding the anatomical details of the temporal loop and provided only schematic illustrations. ${ }^{35,37-40,42}$ The temporal loop is demonstrated in Klingler's work..$^{14-16,21} \mathrm{He}$ alludes to the topic of optic radiation, although this was a secondary focus of his investigations and he discussed it only in relation to the rest of the projection fibers. Recent fiber dissection studies claimed to have demonstrated indi-

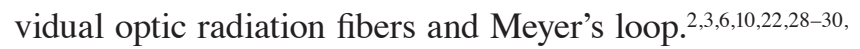
34,44,46,47 The temporal loop is clearly visible in the accompanying illustrations, but it is unclear which of the component fibers in the temporal loop originate precisely in the lateral geniculate body and terminate in the occipital 
visual cortex, because in these illustrations such a course of the temporal loop fibers cannot be completely traced and unequivocally distinguished. Therefore the validity of the anatomical details pertaining to the temporal loop that are derived from these studies remains questionable.

Our fiber dissection examinations on 20 brain specimens and our previous expertise with fiber microdissection of the white matter on more than 200 brains clearly show that a temporal loop of the fibers in the anterior temporal region exists. Contrary to previous assertions, we made a significant observation: all the projection fibers that emerge from the IC-SL, the temporopontine fibers, occipitopontine fibers, and posterior thalamic peduncle (which includes the optic radiation) participate in this temporal loop. In our lateral stepwise dissections, the temporopontine and occipitopontine projection fibers that are located in the lateral aspect of the IC-SL become visible. These projection fibers of the IC-SL continue their course as far as the temporal and occipital cortex by becoming a part of the SS. Our inferior stepwise dissections revealed the roof of the temporal horn covered by the posterior thalamic peduncle, which consists of the projection fibers located in the medial aspect of the IC-SL that originate in the pulvinar of the thalamus and become a component of the SS. The posterior thalamic peduncle incorporates the optic radiation together with the auditory radiation, as well as other temporal and occipital thalamic projection fibers that intermingle and possibly intercross because of their different orientations and cannot be individually differentiated, particularly in the region of the lateral geniculate body.

All these projection fibers of the IC-SL compose the temporal loop and, in their further course, intercross and merge with the fibers of the AC and occipitofrontal fasciculus to become a compact layer, namely the SS. The point of intercrossing that is visible in our dissections is located in the vicinity of the temporal loop of the projection fibers. Beyond the temporal loop the layer of fibers demonstrated in our dissections contains not only the projection fibers but also the commissural and association fibers, which comprise the SS. It is clearly visible in our dissections that the temporal loop is not exclusively composed of the optic radiation but includes all the projection fibers that emerge from IC-SL. We speculate that the existence of a loop of the projection fibers in the anterior temporal region might be related to the embryological development of the brain. The temporal lobe pursues an anterior rotation and forward advancement to attain the definitive position of the temporal pole that entertains a loop of those projection fibers that originate in the subcortical structures, and which then terminate in the more posterior temporal and occipital cortex. ${ }^{27}$

The evidence derived from our fiber microdissection that a temporal loop exists is consistent with the observations of previous studies applying this technique. . $^{2-6,6,10,22}$, 24,28-30,34,44,46,47 Contrary to the assertions in these previous studies, we could not distinguish in this temporal loop between the various projection fibers that emerge from the IC-SL and compose the temporal loop. We could demonstrate with certainty the compact layer of projection fibers in the temporal loop, but it was not possible to irrefutably identify individual components, such as the optic radia- tion, because we were unable to demonstrate fibers that originate exclusively in the lateral geniculate body and to trace such fibers or differentiate them in their further course in the temporal loop. This evidence derived from our fiber microdissection supports the concept that the temporal loop is composed of various projection fibers, including the optic radiation.

The intrinsic limitations of the fiber microdissection technique preclude us from identifying distinctly the optic radiation. Because of these limitations, we believe that what has been defined as the optic radiation in previous studies $^{2,3,6,10,22,28-30,34,44,46,47}$ of fiber dissection may in fact be an oversimplified interpretation of the projection fibers in the temporal and occipital regions and the SS. Although there is only sparse reference to these complex projection fibers, they are visible in these studies' illustrations of the temporal loop. Such an interpretation may have some educational and practical purposes, but we are obliged to maintain our scientific reliability with regard to the complex arrangement of the white matter and respect the limits of fiber dissection investigations when analyzing anatomical observations.

Fiber microdissection is a gross technique that is limited by an inability to trace with precision the origin, termination, and boundary of a certain fiber tract. ${ }^{55}$ The fiber microdissection technique provides a unique, insightful perspective on the complex anatomical features of the white matter that is essential for neurosurgical procedures. The key element offered by applying this technique is a unique, practical appreciation of the anatomical structures, but an awareness of the limitations is necessary. The projection fibers are very thin structures and, even with the highest magnification provided by the operating microscope and the most delicate microtechniques, the fiber microdissection cannot distinguish between the paths of individual fibers. The anatomy of the brain, particularly of the white matter, is far more complex than we are currently able to comprehend. The architecture of the white matter fibers includes intermingling, intercrossing and, possibly, bifurcation of minute axons that take place in a submillimetric space. The fiber dissection technique cannot currently evolve beyond demonstrating and discriminating among gross fiber bundles of millimetric dimensions. Furthermore, a significant amount of anatomical data would be lost in the attempt to dissect and demonstrate individual fiber bundles from this complex structure. Therefore, we concede that the fiber microdissection technique is unable to resolve with accuracy the definite configuration, course, and boundaries of the optic radiation.

\section{Fiber Microdissection Reveals Individual Variations in the Anterior Extent and Angulation Patterns of the Temporal Loop}

Our fiber microdissection examinations revealed significant individual variations in the anterior extension and angulation patterns of the temporal loop (Fig. 4). The temporal loop was located in the anterolateral portion of the roof of the temporal horn, lateral to the tail of the caudate nucleus. The anterior extent of the temporal loop was variable, but never reached as far as the uncal recess or the amygdala. The anteromedial portion of the roof of the 


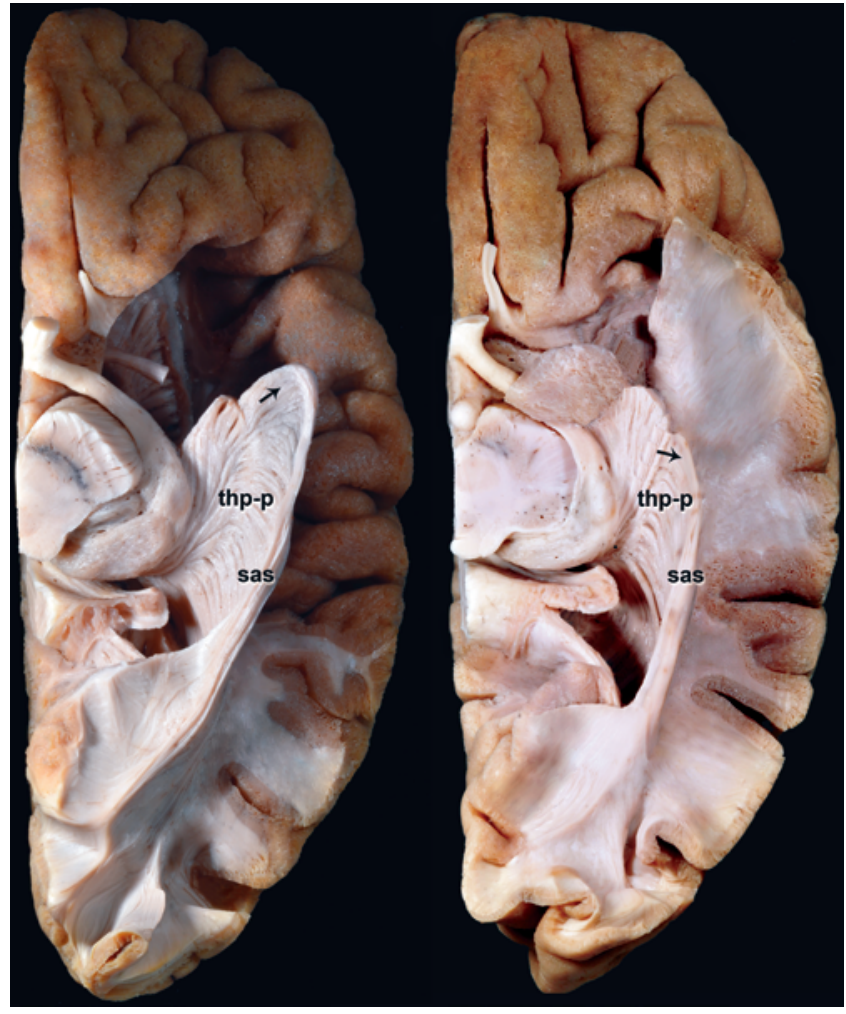

FIG. 4. Photographs of brain specimens showing the inferior aspect of the left cerebral hemispheres dissected to demonstrate the temporal loop of the projection fibers (arrows). The posterior thalamic peduncle (thp-p), which includes the optic radiation, is visible in the roof of the temporal horn of the lateral ventricle. Individual variations of the anterior extent and angulation of the temporal loop of the projection fibers that become a part of the SS (sas) are illustrated.

temporal horn medial to the tail of the caudate nucleus was free of posterior thalamic peduncle fibers, and therefore of the optic radiation.

The precise delineation of the temporal loop eluded us and precluded us from obtaining reliable morphometric data to determine the precise location of this structure, and to quantify the relationship of the temporal loop with key landmarks located on the surface of the temporal lobe. The AC, and the inferior longitudinal fasciculus, on a lateral dissection, and the tapetum, on an inferior dissection, complicate the delineation of the temporal loop. All these fiber systems intertwine and merge with the projection fibers in the temporal loop to form an intricate arrangement in the anterior temporal region. This intricate relationship contributes to the impossible task of distinguishing with accuracy the temporal loop of projection fibers from several other intercrossing fibers, and thus causes inaccurate assessments of the temporal loop's precise anatomical location. The precise location of the temporal loop cannot be determined and quantified by applying the fiber microdissection technique. This limitation of the fiber dissection technique may explain the inconsistent and controversial anatomical quantitative data on the location of the temporal loop, as reported in previous fiber dissection studies. ${ }^{6,28,29,42,46}$

Fiber microdissection is inadequate to accurately delineate the temporal loop from the intricate network of adjoining fibers. Furthermore, because individual optic radiation fibers cannot be distinguished among the various projection fibers of the temporal loop, fiber microdissection is an unsuitable method to elucidate the precise location of the optic radiation fibers in the anterior temporal region. Several investigators support the assertion that the use of fiber microdissection may provide precise and reliable anatomical measurements of the location of the optic radiation, data that can be incorporated into the surgical decision process. . $28,29,46$ This assertion poses potential risks, because it implies that the accuracy of this method has been validated and can be trusted implicitly. Therefore, proceeding with caution is advocated, as is the recommendation to understand the characteristics and limitations of the fiber dissection technique when interpreting anatomical data. This is an especially important issue relevant to Meyer's loop and to the optic radiation, because of the valuable functional role of these structures.

Our fiber microdissection showed clear individual variations, not only in the anterior extension but also in the angulation patterns of the temporal loop. From our observations, the variations in angulation patterns and anterior extensions of the temporal loop might be potentially related to individual morphological characteristics of the cranium. A narrow angulation of the temporal loop may be related to a dolichocephalic morphological type, and a wider, rounder angulation may be a characteristic of a brachycephalic cranium type. A possible method for evaluating our hypothesis may be to correlate the anatomical features of the temporal loop of the projection fibers demonstrated by diffusion tensor fiber tractography, in vivo, with the cranium's dimensions assessed by MRI (Fig. 5). This hypothesis could be further explored following advancements in technology, emergence of high-resolution tractography, and improvements in fiber-tracking algorithms that would discriminate more accurately between projection fibers in the temporal loop and the complex network of adjoining fibers.

\section{Conclusions}

The fiber microdissection technique provides clear evidence to support the definition of the temporal loop as being composed of various projection fibers that course in the IC-SL, rather than only individual optic radiation fibers. The complexity of the white matter fiber network involved in the temporal loop and the significant individual variations form a complex pattern requiring further study and precise analysis. We emphasize the need for further research and advancements in techniques to reveal the complex architecture of the white matter of the brain. Combining fiber microdissection and advanced histological techniques, along with future, higher-resolution fiber tractography, may improve our understanding and prevent misinterpretation of the complex anatomical features of these structures.

\section{Acknowledgments}

We thank Julie Yamamoto for editing the text; Ruben Rodríquez Mena, M.D., for insightful recommendations; and Zeynep Firat, M.Sc., for imaging, image processing, and diffusion tensor fiber 

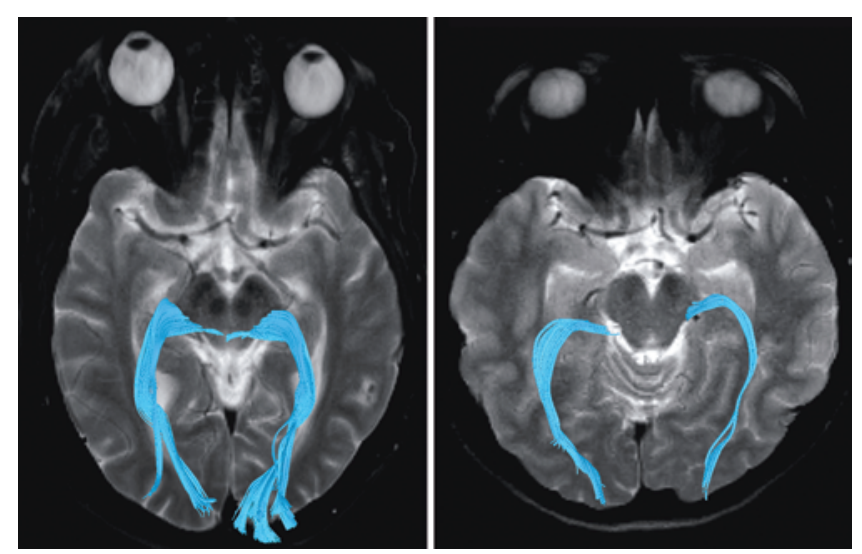

FIG. 5. Diffusion tensor fiber tractography renderings of the temporal loop demonstrating individual variations in the angulation patterns of the temporal loop in 2 previously healthy human volunteers with extreme dolichocephalic and brachycephalic head shapes. Images obtained using a 3T MR unit (Achieva, Philips) with an 8-channel sensitivity encoding head coil. Anatomical morphometric data, derived from a T1-weighted 3D turbo field echo sequence (TR $8.5 \mathrm{msec}$, TE $4 \mathrm{msec}$, slice thickness $1 \mathrm{~mm}$ ), was used to identify the brachycephalic and dolichocephalic types of cranial shape, according to the cranial index. ${ }^{8}$ Diffusion tensor images were acquired using single-shot spin echo planar imaging (TR 10,000 msec, TE $53 \mathrm{msec}$, echo planar imaging factor 67, slice thickness $2.5 \mathrm{~mm}$, gap $0 \mathrm{~mm}$ ). Sixteen diffusion directions at $\mathrm{b}=$ 800 seconds $/ \mathrm{mm}^{2}$ were acquired in addition to $b=0$ images. Sixty slices were taken for whole-brain coverage from the vertex to the foramen magnum. The fiber tracking software (Release 2.6; Achieva, Philips) was used to generate 3D diffusion tensor fiber tractography images. Diffusion tensor tractography imaging of the temporal loop of the posterior thalamic peduncle, which includes the optic radiation, was completed by placing one region of interest in the region of the lateral geniculate nucleus on a sagittal image, and then by placing a second region of interest in the occipital lobe using a coronal image. The 3D reconstructed fibers have been superimposed on axial T2-weighted turbo spin echo (TR $80 \mathrm{msec}$, TE $3000 \mathrm{msec}$, slice thickness $4 \mathrm{~mm}$ ) images to facilitate anatomical orientation. Left: A narrow angulation of the temporal loop demonstrated in a dolichocephalic cranium type. Right: A wide angulation of the temporal loop demonstrated in a brachycephalic cranium type. These images suggest a potential correlation of the angulation of the temporal loop with the shape of the cranium.

tractography analysis. We are grateful to Dianne C. H. Yaşargil for editorial assistance.

\section{References}

1. Archambault L: Le faisceau longitudinal inférieur et le faisceau optique central: quelques considérations sur les fibers d'association du cerveau. Rev Neurol 4:1206, 1906

2. Choi C, Rubino PA, Fernández-Miranda JC, Abe H, Rhoton AL Jr: Meyer's loop and the optic radiations in the transsylvian approach to the mediobasal temporal lobe. Neurosurgery 59 (4 Suppl 2): ONS228-ONS236, 2006

3. Choi CY, Han SR, Yee GT, Lee CH: A understanding of the temporal stem. J Korean Neurosurg Soc 47:365-369, 2010

4. Coppens JR, Mahaney KB, Abdulrauf SI: An anteromedial approach to the temporal horn to avoid injury to the optic radiation fibers and uncinate fasciculus: anatomical and technical note. Neurosurg Focus 18(6B):E3, 2005

5. Cushing H: Distortions of the visual fields in cases of brain tumour: the field defects produced by temporal lobe lesions. Brain 44:371-396, 1922

6. Ebeling U, Reulen HJ: Neurosurgical topography of the op- tic radiation in the temporal lobe. Acta Neurochir (Wien) 92:29-36, 1988

7. Ebeling U, von Cramon D: Topography of the uncinate fascicle and adjacent temporal fiber tracts. Acta Neurochir (Wien) 115:143-148, 1992

8. Eskandary H, Nematollahi-mahani SN, Zangiabadi N: Skull indices in a population collected from computed tomographic scans of patients with head trauma. J Craniofac Surg 20:545-550, 2009

9. Falconer MA, Wilson JL: Visual field changes following anterior temporal lobectomy: their significance in relation to Meyer's loop of the optic radiation. Brain 81:1-14, 1958

10. Fernández-Miranda JC, Rhoton AL Jr, Álvarez-Linera J, Kakizawa Y, Choi C, de Oliveira EP: Three-dimensional microsurgical and tractographic anatomy of the white matter of the human brain. Neurosurgery 62 (6 Suppl 3):989-1028, 2008

11. Flechsig P: Anatomie des Menschlichen Gehirns und Rückenmarks auf Myelogenetischer Grundlage. Leipzig: Thieme, 1920

12. Flechsig P: Weitere mitteilungen über den stabkranz des menschlichen grosshirns. Neurologishes Centralblatt 15:24, 1896

13. Gratiolet PL: Mémoire sur les Plis Cérébraux de l'Homme et des Primates. Paris: Bertrand, 1854

14. Klingler J: Die Makroskopische Anatomie Der Ammonsformation. Zurich: Fretz, 1948

15. Klingler J: Erleichterung der makroskopischen präparation des gehirns durch den gefrierprozess. Schweiz Arch Neurol Psychiatr 36:247-256, 1935

16. Klingler J, Gloor P: The connections of the amygdala and of the anterior temporal cortex in the human brain. J Comp Neurol 115:333-369, 1960

17. Klüver H, Bucy PC: An analysis of certain effects of bilateral temporal lobectomy in the rhesus monkey, with special reference to "psychic blindness." J Psychol 5:33-54, 1938

18. Krieg WJS: Architectonics of Human Cerebral Fiber Systems. Evanston, IL: Brain Books, 1973

19. Krieg WJS: Connections of the Cerebral Cortex. Evanston, IL: Brain Books, 1963

20. Leuret F, Gratiolet P: Anatomie Comparée du système nerveux considéré dans ses rapports avec l'intelligence. Paris: Baillière, 1857

21. Ludwig E, Klingler J: Atlas Cerebri Humani. Basel: Karger, 1956

22. Mahaney KB, Abdulrauf SI: Anatomic relationship of the optic radiations to the atrium of the lateral ventricle: description of a novel entry point to the trigone. Neurosurgery 63 (4 Suppl 2):195-203, 2008

23. Marino R Jr, Rasmussen T: Visual field changes after temporal lobectomy in man. Neurology 18:825-835, 1968

24. Martino J, Vergani F, Robles SG, Duffau H: New insights into the anatomic dissection of the temporal stem with special emphasis on the inferior fronto-occipital fasciculus: implications in surgical approach to left mesiotemporal and temporoinsular structures. Neurosurgery 66 (3 Suppl Operative): $4-12,2010$

25. Meyer A: The connections of the occipital lobes and the present status of the cerebral visual affections. Trans Assoc Am Physicians 22:7-16, 1907

26. Niemeyer P: The transventricular amygdala-hippocampectomy in temporal lobe epilepsy, in Baldwin M, Bailey P (eds): Temporal Lobe Epilepsy. Springfield, IL: Charles C Thomas, 1958 , pp 461-482

27. Nieuwenhuys R, Voogd J, van Huijzen C: The Human Central Nervous System: A Synopsis and Atlas, ed 4. Berlin: Springer-Verlag, 2007

28. Párraga RG, Ribas GC, Welling LC, Alves RV, de Oliveira E: Microsurgical anatomy of the optic radiation and related 
fibers in 3-dimensional images. Neurosurgery 71 (1 Suppl Operative):ons160-ons172, 2012

29. Peltier J, Travers N, Destrieux C, Velut S: Optic radiations: a microsurgical anatomical study. J Neurosurg 105:294-300, 2006

30. Peltier J, Verclytte S, Delmaire C, Pruvo JP, Godefroy O, Le Gars D: Microsurgical anatomy of the temporal stem: clinical relevance and correlations with diffusion tensor imaging fiber tracking. J Neurosurg 112:1033-1038, 2010

31. Peltier J, Verclytte S, Delmaire C, Pruvo JP, Havet E, Le Gars D: Microsurgical anatomy of the anterior commissure: correlations with diffusion tensor imaging fiber tracking and clinical relevance. Neurosurgery 69 (2 Suppl Operative):ons241-ons247, 2011

32. Penfield W, Flanigin H: Surgical therapy of temporal lobe seizures. AMA Arch Neurol Psychiatry 64:491-500, 1950

33. Penfield W, Jasper HH: Epilepsy and Functional Anatomy of the Human Brain. Boston: Little Brown, 1954

34. Peuskens D, van Loon J, Van Calenbergh F, van den Bergh R, Goffin J, Plets C: Anatomy of the anterior temporal lobe and the frontotemporal region demonstrated by fiber dissection. Neurosurgery 55:1174-1184, 2004

35. Pfeiffer RA: Myelogenetisch-Anatomische Untersuchungen uber den Zentralen Abschnitt der Sehleitung. Berlin: Springer, 1925

36. Polyak S: The Vertebrate Visual System: Its Origin, Structure, and Function and its Manifestations in Disease with an Analysis of its Role in the Life of Animals and in the Origin of Man, Preceded by a Historical Review of Investigations of the Eye, and of the Visual Pathways and Centers of the Brain. Chicago: The University of Chicago Press, 1957

37. Probst M: Über die zentralen Sinnesbahnen und die Sinneszentren des menschlichen Gehirnes. Sitz Ber Akad Wiss 115:103-176, 1906

38. Putnam TJ: Studies on the central visual system. II. A comparative study of the form of the geniculostriate visual system of mammals. Arch Neurol Psychiatry 16:285-300, 1926

39. Putnam TJ: Studies on the central visual system. III. The general relationship between the external geniculate body, optic radiation and visual cortex in man: report of two cases. Studies on the central visual system. Arch Neurol Psychiatry 16:566-596, 1926

40. Putnam TJ: Studies on the central visual system. IV. The details of the organization of the geniculo-striate system in man. Arch Neurol Psychiatry 16:683-707, 1926

41. Ranson SW: A description of some dissections of the internal capsule, the corona radiata and the thalamic radiation to the temporal lobe. Arch Neurol Psychiatry 5:361-369, 1921

42. Rasmussen AT: The extent of recurrent geniculo-calcarine fibers (loop of Archambault and Meyer) as demonstrated by gross brain dissection. Anat Rec 85:277-284, 1943
43. Reil JC: Fragmente über die Bildung des kleinen Gehirns im Menschen. Arch Physiol Halle 8:1-58, 1807

44. Rhoton AL Jr: The cerebrum. Anatomy. Neurosurgery 61 (1 Suppl):37-119, 2007

45. Robinson A (ed): Cunningham's Textbook of Anatomy, ed 5. New York: William Wood and Co, 1918

46. Rubino PA, Rhoton AL Jr, Tong X, Oliveira Ed: Three-dimensional relationships of the optic radiation. Neurosurgery 57 (4 Suppl):219-227, 2005

47. Sincoff EH, Tan Y, Abdulrauf SI: White matter fiber dissection of the optic radiations of the temporal lobe and implications for surgical approaches to the temporal horn. J Neurosurg 101:739-746, 2004

48. Standring S (ed): Gray's Anatomy: The Anatomical Basis of Clinical Practice, ed 40. New York: Elsevier, 2008

49. Tecoma ES, Laxer KD, Barbaro NM, Plant GT: Frequency and characteristics of visual field deficits after surgery for mesial temporal sclerosis. Neurology 43:1235-1238, 1993

50. Türe U, Yaşargil DC, Al-Mefty O, Yaşargil MG: Topographic anatomy of the insular region. J Neurosurg 90:720-733, 1999

51. Türe U, Yaşargil MG, Friedman AH, Al-Mefty O: Fiber dissection technique: lateral aspect of the brain. Neurosurgery 47:417-427, 2000

52. Türe U, Yaşargil MG, Pait TG: Is there a superior occipitofrontal fasciculus? A microsurgical anatomic study. Neurosurgery 40:1226-1232, 1997

53. Van Buren JM, Baldwin M: The architecture of the optic radiation in the temporal lobe of man. Brain 81:15-40, 1958

54. Winters E (ed): The Collected Papers of Adolf Meyer. Baltimore: Johns Hopkins Press, 1950

55. Yaşargil MG, Türe U, Yaşargil DC: Impact of temporal lobe surgery. J Neurosurg 101:725-738, 2004

56. Yaşargil MG, Türe U, Yaşargil DC: Surgical anatomy of supratentorial midline lesions. Neurosurg Focus 18(6B):E1, 2005

\section{Author Contributions}

Conception and design: both authors. Acquisition of data: Goga. Analysis and interpretation of data: both authors. Drafting the article: both authors. Critically revising the article: both authors. Reviewed submitted version of manuscript: both authors. Approved the final version of the manuscript on behalf of both authors: Türe. Administrative/technical/material support: both authors. Study supervision: both authors.

\section{Correspondence}

Uğur Türe, Department of Neurosurgery, Yeditepe University School of Medicine, Devlet Yolu Ankara Cad. 102/104 Kozyatagi, Istanbul 34752, Turkey. email: drture@yahoo.com. 